\title{
Families_Share: digital and social innovation for work-life balance
}

\author{
Agostino Cortesi, Carlotta Berionni, Carina Veeckman, Chiara Leonardi, Gianluca Schiavo, \\ Massimo Zancanaro, Marzia Cescon, Maria Sangiuliano, Dimitris Tampakis and \\ Manolis Falelakis
}

(Information about the authors can be found at the end of this article.)
Received 1 February 2021 Revised 12 September 2021 12 November 2021

Accepted 7 December 2021

(c) Agostino Cortesi, Carlotta Berionni, Carina Veeckman, Chiara Leonardi, Gianluca Schiavo, Massimo Zancanaro, Marzia Cescon, Maria

Sangiuliano, Dimitris Tampakis, Manolis Falelakise. Published by Emerald Publishing Limited. This article is published under the Creative Commons Attribution (CC BY 4.0) licence. Anyone may reproduce, distribute, translate and create derivative works of this article (for both commercial and noncommercial purposes), subject to full attribution to the original publication and authors. The full terms of this licence may be seen at http://creativecommons. org/licences/by/4.0/legalcode

This project has received funding from the European Union's Horizon 2020 CAPS Topic: ICT-11-2017, Type of action: IA, Grant agreement No 78078324 (Families_Share) and by MUR FISR 2020 (F2F Families-To-Families for sharing children care)

\begin{abstract}
Purpose - The European H2020 Families_Share project aims at offering a grass-root approach and a codesigned platform supporting families for sharing time and tasks related to childcare, parenting, afterschool and leisure activities and other household tasks. To achieve this objective, the Families_Share project has been built on current practices which are already leveraging on mutual help and support among families, such as Time Banks, Social Streets and self-organizing networks of parents active at the neighbourhood level and seek to harness the potential of ICT networks and mobile technologies to increase the effectiveness of participatory innovation. The aim of this paper is to present and discuss the Families_Share methodology and platform, as well as the results obtained by several partecipating communities in different European countries.

Design/methodology/approach - This paper discusses how the Families Share approach (CAPS project, Horizon 2020) is bringing the sharing economy to childcare. Families Share developed a cocaring approach and a co-designed digital welfare platform to support parents with sharing time and tasks related to childcare, after-school and leisure activities. Families Share conducted two iterative pilot experiments and related socio-economic evaluations in six European cities. More than 3,000 citizens were engaged in the co-design process through their local community organizations and more than 1,700 parents and children actively experimented with the approach by organizing collaborative childcare activities. The authors discuss the challenges and solutions of co-designing a socio-technical approach aimed at facilitating socially innovative childcare models, and how the Families Share approach, based on technology-supported co-production of childcare, may provide a new sustainable welfare model for municipalities and companies with respect to life - work balance.

Findings - The authors discuss the challenges and solutions of co-designing a technological tool aimed at facilitating socially innovative childcare models, and how the Families Share approach may provide a new sustainable welfare model for municipalities and companies with respect to work-life balance.

Originality/value - As a main difference with state-of-the-art proposals, Families_Share is aimed to provide support to networks of parents in the organization of self-managed activities, this way being orthogonal with respect either to social-network functionalities or to supply and demand services. Furthermore, Families_Share has been based on a participative approach for both the ICT platform and the overall structure.
\end{abstract}

Keywords Digital social innovation, Welfare, Work-life balance, Childcare, Co-design, Sharing economy, Socio-economic impact

Paper type Research paper

\section{Introduction}

Balancing work and family life has become increasingly challenging in the past decade all over the world. The economic crisis had a twofold effect: it impacts the labour market conditions as well as welfare provisions. As a result, on the one hand, unemployment rates have risen (mainly in male-dominated sectors), while on the other hand, more women (including mothers) are working on part-time schemes. Stable jobs can no longer be taken 
for granted and precarious contracts are more and more widespread with many workers entering re-qualification schemes and facing unemployment periods.

In Europe, in 2016, only 39\% of children aged less than 12 received formal childcare (paid or not, both public and private). In the majority of member states, the uptake of paid services is higher than the cost-free ones, whereas in other countries, the reasons for not having access to childcare are high costs and/or lack of available places, or suitable opening hours (Eurostat, 2018). After-school activities have also become a luxury that low and medium-income families can rarely afford, leading to impoverished learning and educational opportunities for children (UNICEF Research Office, 2014).

Structural gender inequalities in society, economy and culture impact both formal and informal care work very heavily, which is still largely faced by women. The changing family structures also affect childcare needs as the proportion of mothers of young children in the labour market increases and there is a growing share of single-parent households, because of divorces and diversified living arrangements. Furthermore, an ageing society is posing more constraints, as grandparents tend to be less available to provide childcare support and instead become recipients of care themselves (Fraisse and Escobedo, 2014). At the same time, some members of neighbourhoods and communities often feel isolated and urban milieus' social fabric is made even more complex by their multicultural traits, often ending up in segregation in close ethnic communities.

Within this challenging context, the digital tools that we have available today make new collective intelligence models and participatory methods possible (Ferrario et al., 2014; Manzini and Rizzo, 2011). In our view, smart-city solutions must be based on smartcommunities (De Filippi et al., 2019). To this respect, the concept of "digital social innovation" can support closing the tech-society divide and help reconceptualize technology, and particularly free and open source software, as a means to meet societal needs [Bria et al. (2015)]. Digital social innovation can be an effective way to better cope with the challenges of restricted access to childcare, after-school activities and learning opportunities. The European H2020 Families_Share project [1] aims at offering a grass-root socio-technical approach to support families for sharing time and tasks related to childcare, parenting, after-school and leisure activities and other household tasks. To achieve this objective, the Families_Share project was built on practices which were already leveraging mutual help and support among families, such as Time Banks, Social Streets and selforganizing networks of parents active at the neighbourhood level. Families-Share seeks to harness the potential of ICT networks and mobile technologies to increase the effectiveness of participatory innovation (Dinant et al., 2017; Hillgren et al., 2011). Conscious efforts in communication and engagement strategies were made to prevent project's activities and tools from reproducing existing gender inequalities. In the resulting platform and app, parents can plan and exchange reciprocal childcare, socialization opportunities and informal educational and leisure activities.

This paper provides an overall presentation of the Families_Share platform as well as the results obtained by several participating communities in different European countries. We discuss how this project may contribute towards an integrated local welfare to support work-life balance and gender equality at the municipality and neighbourhood level, and how it may also provide an innovative model for company welfare.

The rest of the paper is structured as follows. Section 2 introduces related state-of-the-art initiatives and solutions on which the project consortium relied and built upon. Section 3 delves into the co-design process that led to the Families_Share platform and mobile app and the various proposed models by Families_Share. Section 4 discusses the validation of the approach within several local communities around Europe. Sections 5 and 6 discuss how the Families_Share approach can provide a new sustainable welfare model for cities, municipalities and companies, respectively. Section 7 discusses the approach from the 
perspective of the socio-economic impacts that were generated and the contribution of the digital platform to those impacts. Section 8 concludes.

\section{State-of-the-art initiatives and solutions}

Families_Share has built on existing evidence and opportunities of integrating bottom-up self-managed informal care fostered by digital tools to support work-life balance. A NESTA study on innovation in childcare pinpoints at specific gaps in childcare provision for parents with atypical work patterns and among those with available local relatives/friends to support. The study argues that informal care often provides the best solution for parents who have ad hoc, emergency and atypical childcare needs, especially if it is supported by digital tools and a bottom-up approach grounded in existing meeting points and networks [Rutter (2016)].

There are not many platforms or digital services specifically aimed at supporting parents in childcare. In the rest of this section, we list a few software solutions that inspired and served as back-to-back references in the design of the Families_Share platform. Bsit [2] provides a supply and demand matching service with the aim of helping families to find trusted babysitters. Similarly, Yoopies [3] is a digital service that supports parents in finding help with their children, starting from the network of friends, acquaintances, family members and work colleagues. It is worth mentioning that none of the existing software systems offer the functionalities required to support self-organized activities while satisfying the nonfunctional requirements (in particular, the ones related to privacy) that are critical when dealing with childcare.

The design of the Families_Share approach was also informed by digital services for supporting time and resource sharing. For instance, Peerby [4] connects peer suppliers with peer consumers: the former offers to rent or share certain items that the latter require. This application works on a neighbourhood basis, and it has been estimated that about 20 people are needed to create sufficient critical mass and to allow sharing to succeed within a particular neighbourhood. Benapp [5] is an Italian platform designed to be a simple, accessible solution that allows people to safely solve everyday problems by sharing their time, talents and resources with the organizations in the area and with their friends. Finally, TimeRepublik [6] is a digital place where the skills and talents of various people could be freely exchanged, without using actual currency as a unit of exchange, but rather time, following the principles of timebanking.

As a main difference with the approaches presented above, Families_Share aims to provide support to networks of parents in the organization of self-managed activities, this way being orthogonal with respect to either social-network functionalities or supply and demand services. Therefore, Families_Share has been based on a participative approach to define a socio-technical framework and not just a digital technology.

\section{Co-designed digital platform for social innovation}

The theoretical stance taken in Families_Share with respect to technology has been that of sociomateriality. It recognizes that the acceptance and the usage of a specific technology is dependent on the institutional and organizational norms in which it takes place, whereas, at the same time, the technology in use affects those institutional and organizational norms (Leonardi, 2012). Our approach has, therefore, been that of designing technology not as a bundle of functionalities but rather as a system of constraints and affordances [Faraj and Azad (2012)] that supports and fosters specific social practices.

The process has been based on the perspective of innovation as the result of co-production and interactions between different local stakeholders and communities involved in the project (Voorberg et al., 2015). Specifically, five innovation labs have been established in 
the cities of Bologna and Venezia in Italy, Györ in Hungary, Kortrijk in Belgium and Thessaloniki in Greece. Furthermore, an additional innovation lab has been established in a medium-size knowledge organization in Trento (Italy).

The use-case studies in the innovation lab were organized as action research interventions in which the researchers actively participated in the activities while also observing their effects (Coghlan, 2019). Because of the socio-technical perspective of Families_Share, the studies have been framed following the grounded design approach (Wulf et al., 2018) to understand the design and appropriation of the service and the role of the digital tool in support of it.

In this context, social innovation has emerged from the negotiation between the requirements expressed by the local communities and the technical (software and legal) constraints and opportunities. In this perspective, the whole approach, and in particular the software platform, is designed and shaped not only throughout the design process, but it is also reshaped through concrete and local use observed during the project pilots (see Figure 1). This process has been grounded on participatory action research (Cargo and Mercer, 2008; Hayes, 2011) using co-design methods (Mulder and Stappers, 2009; Bødker, 2000). Participatory action research is an approach to research in and with communities that emphasize participation and action to understand the world by trying to change it, collaboratively, following reflection and encouraging inclusion (Cargo and Mercer, 2008).

The engagement of stakeholders and potential beneficiaries has been pursued since the very beginning and involved parents, local communities as well as Public Administration representatives, NGO operators, HR managers and other experts. Interviews, meet-ups and surveys were conducted across the six innovation labs to define needs and expectations, as well as to collect best practices, barriers and opportunities. Co-design methods (Bødker, 2000) were used to integrate the different perspectives of the actors and stakeholders involved. In particular, scenario-based design (Carroll, 2000) was used to explore participants' values, and to investigate the opportunities and potentially problematic aspects of a digital platform to support the co-production of childcare.

Overall, around 3,000 people were involved in the workshops and interviews. Three main pillars drove the development of the socio-technical approach:

- The need to combine online and offline to support networks of parents and to guarantee a high level of trust among community members; it was deemed important that people can meet personally and organize face-to-face meetings; this aspect was considered specifically important for ensuring and guaranteeing trust; nevertheless, digital technology may help in providing support to the organization of the activities and the coordination of the group of parents.

- The need to support communities of parents in organizing and coordinating shared childcare activities; digital technology may offer valuable support in the phase of creating groups of parents with a specific goal of organizing an activity; it may also

Figure 1 Co-design and development process of the Families_Share digital platform

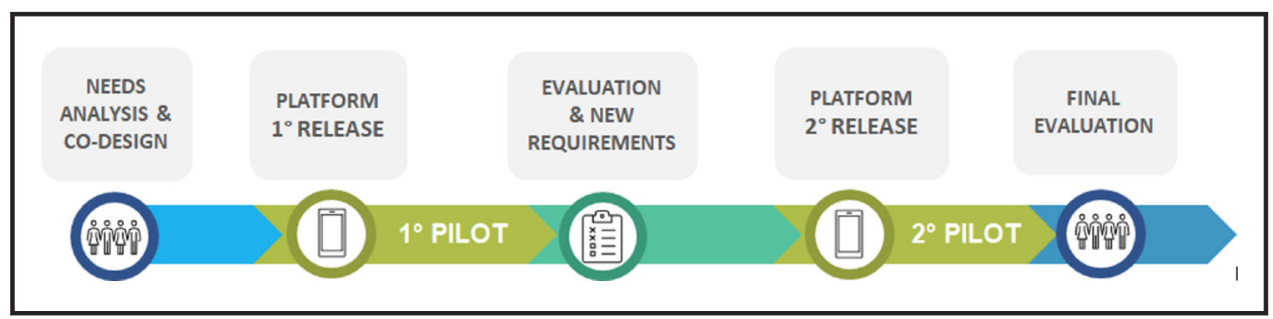

\begin{tabular}{l|l|l} 
VOL. 24 NO. 22022 & DIGITAL POLICY, REGULATION AND GOVERNANCE & PAGE 165
\end{tabular} 
facilitate the coordination among the parents involved in the scheduling and organization of the activities.

- The need to protect privacy and personal data; yet, the overall approach, and specifically the digital technology, should be careful in protecting privacy and personal data of the participants; although some sensitive information is sometimes needed to be shared among participants (for example, about specific needs of some children), it is important that the whole process is controlled and the sensitive information is released on a need-to-know basis.

The Families_Share approach consists in a structured and flexible workflow to support a group of parents to organize a set of self-organized and collaborative activities [7]. Among the various decisions that need to be taken are the rules to regulate how to join the community, how open the activities are and who can organize activities under which conditions.

\subsection{Design pillars for the families_share digital platform}

The specific goal of the Families_Share was to design and deploy a specific digital technology to support and foster collaboration among parents in a specific offline community.

While the co-design activities described above took into consideration the requirements and constraints for the whole process, a specific design effort has been devoted to the digital platform.

The digital platform [8] has the specific purpose of supporting the many varied needs for what concerns group creation and management, but it also supports the many decisions that a group of parents needs to take when organizing a collaborative childcare activity.

Specifically, the platform is composed by a cloud-based backend and a mobile app. The platform supports the several tasks needed to arrange and manage groups of parents working in a collaborative childcare activity: from the initial proposal of the idea, to the collection of interests, up to the actual scheduling of activities and responsibilities and, finally, to the allocation of the specific tasks, including the collection of the specific information on the children involved.

Although the elements of the pillars were common to all the innovation labs, their details and priorities were different. For example, trust was a big concern in the open neighbour-based communities while this was less the case in more strictly organized communities (as the company case study in Trento). Similarly, scheduling was a big issue for large groups that wanted to organize long-term events, such as summer camps; it was less of a concern for small groups organizing ad hoc activities.

From a technical point of view, multiple instances of the Families_Share Web app were deployed, creating smaller and on-demand instances. Each Web app instance is deployed in an isolated container with its own database. This ensures that data is not shared among different communities and it also allows customization of the functionalities described above to better fit each single community.

Even if all instances originate from the same codebase and share the same core functionalities, each Families_Share community may have characteristics that differentiate it from the other ones: from a strict control on new user registrations, to presence/absence of some functionalities.

For example, in some innovation labs, one of the most important features that the platform was designed to offer is an advanced planning functionality, which helps parents in finding optimal scheduling solutions (Figure 2). This functionality aims to automate the activity creation and participation process, by taking several factors into account and producing an optimal plan of scheduled activities with fair distribution of members' participation. For some 
Figure 2 Screens from the Families_Share mobile application: left, the groups and activity screen with information on the childcare activities organized within each group; and, right, the advanced planning functionality that support parents in finding optimal scheduling solutions for the activities

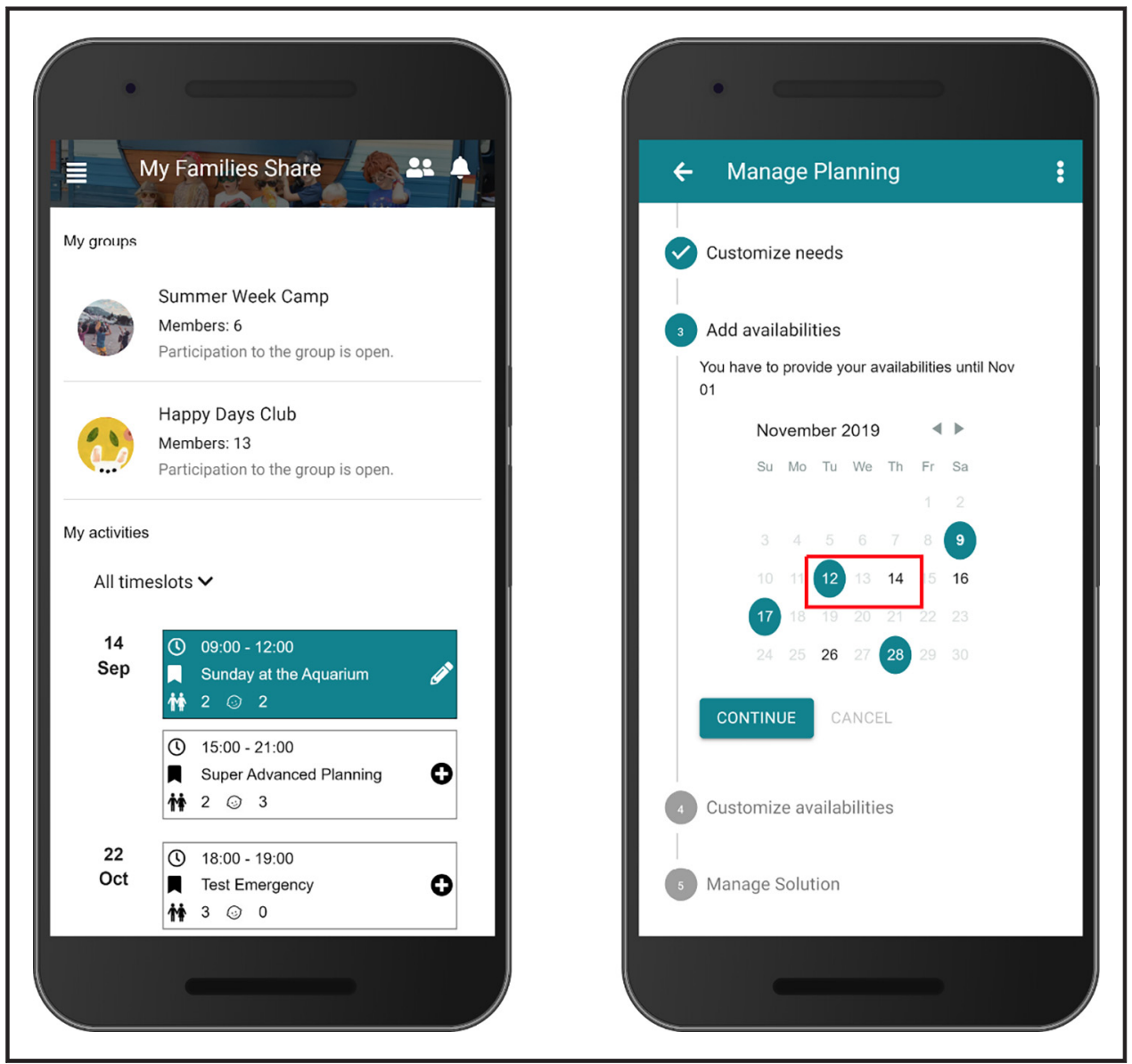

communities, this functionality was not made available and replaced with a more limited but much simpler calendar.

While the Families_Share mobile application included several features of both a social network and a management tool, it is neither one nor the other. Its design has been based on the specific needs of families and values of parents, and it has been intentionally conceived to support and foster offline activities.

\section{Type of models and pilots of Families_Share}

From the second half of 2018 until summer 2020, more than 1,700 parents and children (age 3-10) were actively experimenting with self-organized and collaborative childcare activities using the Familie_Share approach in the six innovation labs, among which five of them were neighbourhood-based, while one was a based on a company welfare intervention (Trento). Community managers from the project facilitated the groups of parents to self-organize the activities in shifts (named co-playing) typically with 10-12 children; these activities were always supervised by at least two adults.

A thorough evaluation process, with qualitative research methods (Patton, 2014; Silverman, 2016), accompanied the whole process in the two years of piloting: 
participant observations were conducted during the entire period and focus groups and individual semi-structured interviews with participating parents were conducted at the end of each iterative period.

Two major types of co-playing activities emerged in the pilot communities: co-playing weeks during holidays (e.g. summer, Christmas, spring holidays, etc.) and single afterschool or afternoon activities. Co-playing weeks constitute a more straightforward choice prioritizing the goal of offering a cheap and affordable solution to childcare during holiday periods and require more volunteering efforts from the parents' side. For co-playing weeks, an advanced planning functionality of the platform proved to meet parents' needs for availability matching, especially in larger and more experienced communities such as Kortrijk where many of the groups of parents set up and manage 2-3 weeks per year each group.

Also, co-playing models varied, combining informal and volunteer caring-time from parents and formal, paid caring time from professional educators in different ways:

- Fully self-organization by parents (that is, a model relying exclusively on informal care): this implies parents being in charge of the activities entirely, so the shifts' scheduling is more complex, the workload higher as well as the creativity and caring skills needed to plan and set up games or other activities for the children.

- Mixed approach: combining informal and formal care; this model implies a more or less constant presence of professional educators during the activities most of the time with a leading role, and with parents taking more supportive functions; this model was more manageable in terms of efforts required to parents, while the costs for hiring educators was managed in different possible ways: in some cases, it was shared by parents; in others, it was part of a fee for a formal care solutions to whom the parents got discounts for making their time available.

Within the project, these models were taken up in different ways: some of the communities opted for the mixed model (this was the case in Trento, Bologna and Györ), and others relied on an entirely self-organized care-sharing model (Kortrijk) or piloted both models (Venice). To integrate the participation of external educators in the activities, an "external educator" section was also added as a functionality in the app to support filling some slots with paid babysitters or caregivers. For the after-school activities use-case, and the mixedmodel approach in general, we observed the need of building trust and communities of parents was less crucial when compared to co-playing weeks use-cases, and entirely selforganized model approach: the more the activities required efforts and active role from parents over longer periods, the more mutual trust was needed in the groups. Furthermore, it is important to note that the digital platform was not by itself the driving force of a trustbuilding process: the digital component may support groups of parents that already belong to the same "network of trust", and thus remains a means to support and facilitate a trustbased social innovation process.

Another relevant aspect to note is that, although women represented the majority of parents, targeted communication campaigns and engagement activities allowed to shift from an initial $11 \%$ of involved fathers to $25 \%$ during the last round of activities in the Summer and Autumn 2020.

Finally, during the pilot experiments, the Covid-19 outbreak had a great impact on the Families_Share activities. During the lockdown period, most communities were able to reorganize and hold online activities to continue supporting parents and children, such as homework support, cooking or scientific laboratories and origami, info sessions and talks, in a continuous mutual learning approach. In this case, the digital platform was mainly used to schedule the meetings and to create closed groups per neighbourhood or per activity, so that families could meet and get to know each other first. 


\section{Towards integrated local welfare to support work-life balance through self- organized care and a mixed-model approach}

This section provides a reflection on the three main aspects that emerged from the experiences at the municipal and neighbourhood levels (Venice, Bologna, Thessaloniki, Gyor and Kortrijk), namely, trust and social homogeneity, access to public spaces for organizing activities and gender equality issues. Overall, we learnt that, if such issues are taken into account and mediated at the local level, a digitally supported social innovation such as Families_Share has the potential to contribute to a more sustainable integrated local welfare to address work-life balance (Andreotti and Mingione, 2014). To this respect, our findings confirm recent studies on the dynamic and contextual nature of social innovation processes which tend to evolve towards co-creation processes (Bartels, 2017; Voorberg et al., 2015).

Community building and socialized parenting were valued by parents in all innovation labs as key positive elements in their participation in Families_Share activities. Still, for this to happen, trust was a needed pre-requisite. In fact, especially being a first experience for almost all, parents expressed concerns about their own capacities in being responsible for co-playing with small groups of children, and they needed time to fully trust other parents as well. The project pilot experiments showed how trust can be sustained and enhanced in two main ways: either the informal and self-managed trait of the shared childcare experience is balanced by the presence of mediating organizations or professionals (institutional/formal childcare providers) and bends towards formal childcare, or for the entirely self-organized model, group homogeneity becomes the leverage for trust and parents feel more at ease both entrusting their children to others and co-organizing playing and creative activities.

The piloting experience indicated how parents appreciated the possibility to combine their shared time and volunteer childcare with support from professional educators (the "mixed model"). This seemed to work well both for overcoming resistance to the proposed social innovation enhancing the overall trust in a new practice such as shared childcare, and more practically, for reducing the time parents had to make themselves available. This was the case especially for full-time working parents and for longer schedules such as co-playing weeks during summer or winter holidays. In fact, in our pilot research, entirely selforganized schemes where parents were entirely in charge of managing activities were adopted by the most homogeneous groups. This was the case in the Venice island, a highly gentrified context where the prevailing socio-economic profile of participants was made of highly educated professionals, with flexible jobs and public administration employees. Also in Kortrijk, especially during the first years of piloting, co-playing parents were mostly belonging to the same schools/kindergartens community, so-called "method-schools", based on child-centred, outdoor education and already relying on parents' active engagement, and typically attended by middle class native families.

According to the results of the project, there is space for an active role for both NGOs (mostly those already active in childcare and educational activities) and local municipal authorities or public schools to sustain and be key players in integrating practices such as Families_Share in their "traditional" formal childcare/welfare offer. In fact, the Families_Share innovation labs were either integrated within an already existing childcare and summer camp facility run by NGOs (i.e. in Gyor), or directly run by NGOs having co-playing as their core mission activity (in Kortrijk and Venice). Also, in Thessaloniki, Venice and Bologna, several NGOs took part in the process, making their own professional educators and/or volunteers available to support parents in the activities. In all the innovation labs, partnership and collaboration with cities and local authorities were proven to be important in many respects. In Venice, for example, municipal pedagogists provided advice to parents on how to better handle educational and leisure activities for small groups of children, and the municipality integrated Families_Share in the official summer camps' offer.

VOL. 24 NO. 22022 DIGITAL POLICY, REGULATION AND GOVERNANCE $\mid$ PAGE 169 
In Venice, Bologna and Kortijk, families relied on city authorities or on public schools to have access to spaces where co-playing activities were hosted: in such cases, the practice made it possible to form groups of parents or caregivers and to make use of public urban commons (schools, kindergartens, play-centres), in times when they were under-used. Regulatory issues had to be addressed and solved based on the local frameworks, which were found to be not fully apt for facilitating access and use of public spaces from citizens and bottom-up initiatives, as already reported by many scholars (laione, 2016; Madanipour et al., 2013), making the overall experience more complex and burdensome [9].

Another "thorny issue" addressed by Families_Share was what we referred to as the (gendered) paradoxes and tensions between formal and informal care work. In fact, because the Families_Share model is based on parents' volunteer childcare and mutual support, it brings part of the childcare work out of the formal care and "services" realm, to informal nonpaid care. This can generate and has generated a series of dynamics. On the one hand, we could look at self-managed and volunteer childcare based on mutual help as forms of reappropriation of reproductive labour and re-configuring the productive/re-productive labour relation (Federici, 2012). In fact, many of the participating parents, fathers in particular, opened up time for care within their routine schedules often dominated by productive work, and many mothers reported they enjoyed the opportunity of overcoming isolation and socializing childcare. On the other hand, in the majority of cases, participation in the co-playing activities still was not equal, as women were still the vast majority of co-playing parents.

A wider uptake of an entirely self-organized and self-managed model appeared to have intrinsic limitations for full-time working couples [10] while being more viable in two-parents families and when one of the two was working part-time (most often women), or unemployed. This led us to consider how a widespread diffusion of the entirely selforganized model in contexts featured by strong gender inequalities in employment patterns could risk exacerbating them, unless mitigating supporting/measures are put in place.

Overall, as recalled above, the evaluation showed that the project's invested efforts in engaging fathers paid off, and led to a higher participation rate from $11 \%$ to $25 \%$ in the coplaying activities, compared to an average $5 \%$ in formal childcare staff.

Thus, providing incentives for male participation and sustaining women to improve their professional/working situation would be useful compensation measures, should the informal, entirely self-organized and social innovation-based share of childcare activities increase.

Finally, in line with the risks emphasized by literature on social innovation (Cels et al., 2012; Verhoeven and Tonkens, 2013), concerns were mostly raised by networked stakeholders and NGOs, that initiatives such as Families_Share could lead (local) public authorities to disengage and cut budgets for publicly funded childcare, or that they compete with private and formal care services, which again are often run by and/or mostly employ women. In this respect, the project's results seem to indicate that the share of informal childcare activities can complement rather than substitute the public formal childcare offer. A mixed-model approach, where families collaborate with local NGOs already offering childcare services and the active engagement of public authorities, could lessen the above-mentioned tensions.

In this respect, policy lessons can be drawn from the project, as even if strongly relying on volunteering time from families and non-governmental organizations, the role to be played by local public authorities and/or companies is crucial for the success of the initiatives. Public welfare/childcare policies could benefit from opening spaces to co-creative models that integrate social innovation also as a way to reinforce solidarity in neighbourhoods. In such a framework, municipal administrations should act as mediators to facilitate families and the involved NGOs in the process in accessing important resources such as spaces and schools/ kindergartens in particular during holiday seasons when they are not used. To this regard, in most of the cities and countries where Families_Share was piloted, issues were faced because of existing regulatory framework not allowing informal groups of citizens to access and use 
spaces owned by local authorities, because of lack of insurance coverage in such cases. In Bologna, only the city had a formal procedure in place to make this possible, in the form of "collaboration pacts" known as a good practice for the use and reuse of "urban public goods" by citizens [11]. Also, as it happened in Venice, for example, local authorities could integrate models such as Families_Share in the regular offer of publicly supported summer/holiday camps; they could make resources such as communication to reach out to families or training tools for engaged parents on running educational activities for kids by involving internal staff. The role of the public could entail as well networking among local NGOs to facilitate synergies and mutual help and, last but not least, it would be important for monitoring the gender-related risks highlighted above, so to provide unemployed or part-time employed women involved in the activities with support to further qualify and strengthen their professional opportunities. Based on results from the project, it appeared as favourable public policy frameworks are needed for the success of such practices in company settings too: in fact, the Families_Share pilots took place successfully in a context featured by proactive public welfare policies where regional authorities encourage and concretely support through a variety of measures public and private employers to innovate and facilitate work-life balance of their staff.

\section{Families_Share as a model for company welfare}

Recently, extensive research suggests that an organizational culture supporting and valuing the integration of employees' work and family lives reduces work-family interference (French and Shockley, 2020; Koekemoer and Petrou, 2019; French et al., 2018; Lapierre et al., 2018). Work-life balance is positively related to job satisfaction and affective commitment (Haar et al., 2014; Anafarta, 2011) and organization performance (Wong et al., 2020). Consequently, organizations often establish family-friendly policies (French et al., 2018; Lapierre et al., 2018) through formal support (in the form of time, finance or services) and informal support (in the form of material or psychological resources through social relationships). The extant literature suggests that informal supports are more effective than formal ones (French and Shockley, 2020).

As part of the Families_Share activities, the Trento innovation lab focused on exploring how the Families_Share approach could be implemented in an organizational setting as part of other informal supports to work-life balance (Leonardi et al., 2011, 2019; Schiavo et al., 2020). The study was conducted within a medium-size knowledge-based organization with almost 400 employees based in North Italy. Knowledge-based organizations are characterized by flexible working time arrangements and short-term work contracts and are considered a key pillar of today's knowledge economies (Sousa and Dierendonck, 2010).

The case study is briefly summarized here, while more details are reported elsewhere (Casula et al., 2020; Schiavo et al., 2020). As a first step, institutional stakeholders and employees were involved to get an understanding of their attitude towards collaborative forms of childcare. Perceived values and potential barriers of social and organizational arrangements were identified. In particular, we recognized the mediating role of interpersonal trust, social exchange and reciprocity. This initial evidence was used to drive the platform's design (see above) and, in collaboration with the HR department, the definition of the activities implemented in the second step of the action research.

Two different forms of collaborative on-site childcare initiatives were activated: one-weeklong full time educational and recreational activities organized for employees' children during the summer school break (called Summer Labs) and after-school activities hosted in a specific dedicated room at the organization's premises and during the working hours on Friday afternoon (called Afternoon Labs). They both involved employees in the coproduction of the service but to different extents. The Summer Labs were characterized by a general top-down approach from the organization's management to the employees. They were based on a mixed collaboration between external professionals and employees' involvement in proposing scientific activities or supporting more mundane activities such as

\begin{tabular}{l|l|l} 
VOL. 24 NO. 22022 & DIGITAL POLICY, REGULATION AND GOVERNANCE & PAGE 171
\end{tabular} 
serving food. The Afternoon Labs tried to foster a complete bottom-up collaborative governance by employees with supervision and support by HR management.

The general response was positive as the employees appreciated the opportunity of blending work and family life by being involved in a community of co-working parents and actively participating in childcare activities. Nevertheless, participation was felt to be too demanding in terms of time, effort and emotional involvement for the employees. In line with results from the neighbourhood-based innovation labs, eventually, the mixed model experimented with the Summer labs, which balances external professional competencies in childcare with limited employee involvement, seemed to maximize the benefits and minimize the drawbacks. Furthermore, some volunteering parents felt that the organization did not properly recognize their participation. Although participation was indeed taking place within working hours, some participants stressed that this aspect should be formalized in the organization's internal regulation.

For what concerns the organization's point of view, collaborative childcare was recognized by HR managers, as well as by organizational governance, as an opportunity to implement informal work-life balance initiatives. Nevertheless, some concerns were also raised. The first aspect regards the cost; these types of initiatives are more expensive than the typical work-life benefits offered by companies, in particular, when the time volunteered by the employee is appropriately accounted for. Furthermore, HR managers noted that staff with working shifts and employees in front-end services have more difficulty participating in collaborative childcare services, resulting in unfair access to this opportunity inside the organization. Finally, another perceived barrier was the difficulties in communicating opportunities to employees and quickly assessing their needs in terms of work-life balance: this aspect may represent a main critical feature for employee engagement and a proper mapping of employee's needs.

For what concerns the technological platform, it was considered useful for engaging employees for both communication and organization of work. Yet, the actual use of the platform by HR employees was quite limited, mainly because of the lack of integration in their process and existing IT infrastructure.

Inconclusion, the Trento case study demonstrated that collaborative childcare in the workplace might be a promising opportunity to implement informal support to work-family balances. Still, it is essential to consider the potential drawbacks by careful and considerate planning by the HR department. Nevertheless, it may offer an opportunity for collaborative engagement to a more outstanding work-life balance and foster innovative forms of public-private partnerships for childcaring as envisaged by a recent report of the European Commission (Barcevičius et al., 2020).

\section{Socio-economic impacts of the Families_Share approach and the role of the digital platform}

During the project lifetime, surveys, interviews and focus groups were organized with participants in the innovation labs. These evaluation activities were set up to measure the socio-economic impacts of the Families_Share approach. For conducting the socioeconomic impact assessment, a specific monitoring instrument was set up for evaluating the outcomes and impacts of the Families_share solution. The applied evaluation framework, consisting of different impact indicators, monitored and analyzed different operational levels (micro, meso and macro operations) and with different stakeholders of the project [12]. This framework was tailored to the social innovation goals of the Families_Share project, whereby impacts were conceptualized as the benefits towards the communities and society as a whole, which result from the project outcomes (Epstein and McFarlan, 2011). The monitored impact indicators were as follows:

- parent well-being;

- child well-being; 
- community building; and

- social capital.

The economic impact indicators were as follows:

- economic empowerment; and

- employability.

These indicators were monitored and evaluated across the three different models of Families_Share.

\subsection{Social impact}

The assessment proved that the well-being of parents improved, thanks to the Families_Share solution. The well-being of parents was measured through the perceived impact of Families_Share on the work-family balance, the level of stress because of arranging childcare and on time saved (for leisure time). A total of $64 \%$ of participating parents agreed that their work-life balance improved and $57 \%$ of the parents felt less stressed. Parents perceived Families_Share as a well-fitting alternative childcare solution with well-planned activities. The children were enjoying the activities and were eager to participate as they took place in a familiar environment. This caused a relief from stress (both for children and for the parents), compared to unfamiliar summer camps.

Next, in terms of child well-being, almost all parents mentioned a very positive impact and reported positive experienced emotions of their children, and most of them reported feeling "excited", "enthusiastic" and "crazy" after attending a Families_Share activity. Other important indicators of children's well-being were the influence on social development skills. In the assessment, $70 \%$ of the parents affirmed that Families_Share helped in improving the social development skills of their children, such as communication, collaboration or socializing skills and that it enriched the upbringing context of their children. Parents reported that their children learned how to plan activities; adhered to decisions taken together with the supervising adults; engaged in taking care of younger children in the group; and learnt to better relate with and trust other parents in the group. Last, children's relationship with their parents was also positively affected by their participation in Families_Share activities. Most children felt extremely happy when their parents are co-supervising the group and it also resulted in spending more time together with their children (e.g. preparing the activities together).

In terms of community building, Families_Share succeeded in creating trust among other parents or guardians to take care of their children. Almost 93\% reported a moderate to great impact of their participation in Families_Share on their feeling of trust towards other parents. In a similar vein, $67 \%$ reported a moderate to great impact on their feeling of comfort towards sharing personal information (about themselves and their children). In the case of school community-based co-play groups, it allowed parents to know each other better and bond, and to feel more connected to each other. Further, the parents expressed that they enjoyed being part of a democratic, collaborative and respectful community, where every parent can express their opinion and participate actively in decision-making.

The last social impact indicator focused on social capital, which measured the impact on group management skills and activity management skills. A total of $63 \%$ of the parents reported a positive impact on their group and activity management skills through their participation in the childcare sharing activities. Parents reported learning how to be more creative in the co-play group activities and also improved their self-confidence for arranging childcare tasks. Many parents managed to facilitate large groups of children, and knowledge and skills related to childcare were transferred among the parents (e.g. complementing each other's group tasks). 


\subsection{Economic impact}

Besides social impact indicators, the economic impact of Families_Share was also monitored and evaluated. A total of $27 \%$ of the engaged parents did report that because of the saved time, thanks to Families_Share, they were able to work more hours.

Furthermore, in terms of economic value gain, $71 \%$ of the parents reported that Families_Share allowed them to save costs from paid childcare. The low financial cost of participating in Families_Share, compared to other childcare options, influenced positively the participation of many parents in the co-play group activities. Last, $50 \%$ of the parents also reported sharing goods among the members of the community (e.g. clothes, toys, etc.). These findings indicate that the Families_Share approach has a positive influence on the sharing economy and also leads to monetary benefits for some of the parents.

\subsection{Assessing the impact of the digital platform}

The usability of the platform, as measured throughout the project, was relatively high. Parents did not have problems in understanding and using the functionalities. In particular, they have appreciated it for those tasks that are more difficult to perform with other tools, such as, for example, the complex scheduling needed for managing the longer activities in the Venice's innovation lab. The usability was also improved by the possibility (offered to the organizers) to customize the functionalities of the mobile application to the actual needs of the community (for example, the helpful but complex scheduling functionality was not offered to the smaller groups in Trento's innovation lab).

Nevertheless, as noted above, in some cases, the actual usage also depended by the specific digital maturity of the people involved. In particular, in the organizational contexts, the HR managers complained that the digital platform was not integrated in their workflow.

\section{Conclusion}

In this paper, we presented the foundations and evaluation results of the Families_Share approach for self-organized and collaborative childcare. The approach consists of a bottomup organized process of care and a software platform co-designed with over 3,000 people in Europe in six different innovation labs located in four countries in Europe. The most important lesson learned for the relevant stakeholders is that, albeit the same critical aspects emerged in each innovation labs, the specific instances were completely different. Therefore, it is of paramount importance to identify how the important notions of trust, privacy, security as well as the technical aspects of scheduling, communication and agreement are instantiated in each individual community. The Families_Share approach is focused on investigating parents' and societal needs, developing flexible bottom-up solutions and a dedicated platform, evaluating the activities carried out, while integrating feedback and opinions in a continuous digital-social innovation process. In this respect, digital technology is an adaptable tool to implement this approach rather than a way to structure it (Tchounikine, 2017).

An important lesson learned from the project concerns the need to incrementally build the trust in the process. Local communities need to learn how to autonomously evolve their understanding of collaborative childcare. While in some of our innovation labs, the model relying on parents being entirely self-organized has been fully embraced, others prefer a milder but more manageable mixed model by involving professional third parties. Again, the possibility to customize and adapt the approach is crucial for its adoption, and attention is needed to balance socio-cultural homogeneity as a leverage of trust, particularly with entirely self-organized groups. Further research and action is needed to fully understand the necessary adaptation of the practices and tools to facilitate the involvement of most vulnerable populations, as people with lowest income and migrants were a minority of the participating families: from preliminary observations, difficulties in fully adhering to the balanced time sharing and to the use of the app can emerge, which deserve more in-depth study. 
Other key learning aspects for Families_Share concern the relation with "traditional" formal childcare/welfare and gender implications of shared-care models. The experiences in the innovation labs have demonstrated that an integration of the two models is the key to a successful implementation of effective childcare initiatives with parents undertaking an active role. In some cases, the integration is driven by the grassroot initiatives (like for the case of Bologna and Kortrijk) with support from the public services; in other cases (such as Venezia and Gyor), the role of NGOs was crucial.

With regard to gender implications, it clearly appeared, as expected, that unless specific efforts are undertaken to address and engage with fathers, structural gender inequalities in childcare tend to be reproduced also in informal and bottom up settings, leading to femaledominated groups. Also in both models and whereas (unemployed, part time) women are prevailing, the risk that this becomes for them an additional negative incentive towards employment should be taken into account and mitigation measures set in place. Finally, in the case of Trento, the formal role of an organization was the key to trigger and support collaboration (among employees): although the process was driven from the top, the selforganizing component was present and crucial to the success of the initiative.

The digital platform proved to be a valuable support to the collaboration, in particular for the more complex situations and tasks. Nevertheless, it is worth noting that a digital technology may aim to foster and support new practices but it can never succeed alone. In this respect, the relative success of the Families_Share approach is because of the combined design of both the technology and the service together.

The Families_Share approach is openly available in the form of a multilingual toolkit and an open source app (see above). The overall positive perception from external interested stakeholders has already led to replication in different cities and contexts. Although our work was grounded in existing literature on digital and social innovation processes and governance, we believe that the experience of social innovation in childcare, both in local neighbourhoods and in company settings, described here may shed new light on this important topic and may promote a better gender equal and integrated welfare system for work-life balance with various social economic impacts.

\section{Notes}

1. Families_Share project website: www.families-share.eu.

2. Bsit: https://bsit.com/

3. Yoopies: https://yoopies.com/

4. Peerby: www.peerby.com/

5. Benapp: www.facebook.com/benappCommunity/

6. TimeRepublik: www.timerepublik.com

7. The Families_Share approach is available as a toolkit in different languages here: www.familiesshare-toolkit.eu/

8. The platform is deployed under an open-source license (Apache license 2.0), as a non-exclusive, no-charge, royalty-free, irrevocable copyright license to reproduce and distribute; open-source code is available as a Github repository in https://github.com/vilabs/Families_Share-platform

9. Insurance and liability issues were found representing the bottleneck of entrusting informal groups of citizens to make use of public spaces, and this in turn tends to make the creation of legal entities/NGOs a necessity, while at the same time, ending up "institutionalizing" bottom-up social innovation practices and, more concretely, adding an extra burden of paper and organizational work on parents themselves (and additional liability on those who accept being in the governing bodies of the NGOs).

10. For full-time working parents, three to four full days of volunteering time, subtracted to vacation time, allows families to enroll their kids in 2.5 summer or winter holidays weeks approximately, far less than the school holidays average. 
11. Comune di Bologna, Regulation on collaboration between citizens and the City for the care and regeneration of urban commons. www.comune.bologna.it/media/files/bolognaregulation.pdf

12. This framework relied on the previous work of the IA4SI project (Passani et al., 2016). This latter project was a coordination and support action and built a framework in the context of Collective Awareness Platforms for Sustainability and Social Innovation (CAPS) to measure social, economic, environmental and political outcomes and impacts.

\section{References}

Anafarta, N. (2011), "The relationship between work- family conflict and job satisfaction: a structural equation modeling (SEM) approach", International Journal of Business and Management, Vol. 6 No. 4, pp. 168-177.

Andreotti, A. and Mingione, E. (2014), "Local welfare systems in Europe and the economic crisis", European Urban and Regional Studies, Vol. 23 No. 3, pp. 252-266, doi: 10.1177/0969776414557191.

Barcevičius, E., Codagnone, C., Klimavičiūtè, L. and Misuraca, G. (2020), "Exploring digital government transformation in the EU: expert consultation and stakeholder engagement (no. JRC121494)".

Bartels, K. (2017), "The double bind of social innovation: relational dynamics of change in neighbourhood governance”, Urban Studies, Vol. 54 No. 16, pp. 3789-3805.

Bødker, S. (2000), "Scenarios in user-centred design - setting the stage for reflection and action", Interacting with Computers, Vol. 13 No. 1, pp. 61-75.

Bria, F., Gascò, M. and Kresin, F. (2015), "Growing a digital social innovation ecosystem for Europe". NESTA, report commissioned by EC-DG Connect.

Cargo, M. and Mercer, S.L. (2008), "The value and challenges of participatory research: strengthening its practice", Annual Review of Public Health, Vol. 29 No. 1, pp. 325-350.

Carroll, J.M. (2000), "Five reasons for scenario-based design", Interacting with Computers, Vol. 13 No. 1, pp. 43-60.

Casula, M., Leonardi, C. and Zancanaro, M. (2020), "How does digital technology impact on the coproduction of local services? Evidence from a childcare experience", Public Money \& Management, Vol. 42 No. 2, pp. 87-97.

Cels, S., De Jong, J. and Nauta, F. (2012), Agents of Change: Strategy and Tactics for Social Innovation, Brookings Institution Press, Washington, DC.

Coghlan, D. (2019), Doing Action Research in Your Own Organization, SAGE Publications, UK.

De Filippi, F., Coscia, C. and Guido, R. (2019), "From smart-cities to smart-communities: how can we evaluate the impacts of innovation and inclusive processes in urban context?", International Journal of E-Planning Research (Research), Vol. 8 No. 2, pp. 24-44.

Dinant, I., Floch, J., Vilarinho, T. and Oliveira, M. (2017), "Designing a digital social innovation platform: from case studies to concepts", International Conference on Internet Science, pp. 101-118.

Epstein, M.J. and McFarlan, F.W. (2011), "Measuring the efficiency and effectiveness of a nonprofit's performance", Strategic Finance, Vol. 93 No. 4, p. 27.

Eurostat. (2018), "Access to social services. Almost 4 in 10 children in the EU receive formal childcare services", Eurostat News Release 30/2018, available at: https://ec.europa.eu/eurostat/documents/ 2995521/8681785/3-20022018-AP-EN.pdf/59fcfaa7-0c72-48a6-8603-899b5b730773

Faraj, S. and Azad, B. (2012), "The materiality of technology: an affordance perspective", in Leonardi, P.M., Nardi, B.A. and Kallinikos, J. (Eds), Materiality and Organizing, Oxford University Press, UK, pp. 237-258.

Federici, S. (2012), Revolution at Point Zero. Housework, Reproduction, and Feminist Struggle, PM Press, Brooklyn.

Ferrario, M.A., Simm, W., Newman, P., Forshaw, S. and Whittle, J. (2014), "'Software engineering for'social good': integrating action research, participatory design, and agile development", in Companion Proceedings of the 36th International Conference on Software Engineering, Changing family needs and local childcare policies, pp. 520-523.

Fraisse, L. and Escobedo, A. (2014), Social Vulnerability in European Cities, Springer, Germany.

French, K.A. and Shockley, K.M. (2020), "Formal and informal supports for managing work and family", Current Directions in Psychological Science, Vol. 29 No. 2, pp. 207-216.

French, K.A., Dumani, S., Allen, T.D. and Shockley, K.M. (2018), "A meta-analysis of work-family conflict and social support", Psychological Bulletin, Vol. 144 No. 3, pp. 284-314. 
Haar, J.M., Russo, M., Suñe, A. and Ollier-Malaterre, A. (2014), "Outcomes of work-life balance on job satisfaction, life satisfaction and mental health: a study across seven cultures", Journal of Vocational Behavior, Vol. 85 No. 3, pp. 361-373.

Hayes, G.R. (2011), "The relationship of action research to human-computer interaction", ACM Transactions on Computer-Human Interaction (Interaction), Vol. 18 No. 3, pp. 1-20.

Hillgren, P.A., Seravalli, A. and Emilson, A. (2011), "Prototyping and infrastructuring in design for social innovation", CoDesign, Vol. 7 Nos 3/4, pp. 169-183.

laione, C. (2016), "The CO-city: sharing, collaborating, cooperating, and commoning in the city", American Journal of Economics and Sociology, Vol. 75 No. 2, pp. 415-455.

Koekemoer, E. and Petrou, M. (2019), "Positive psychological interventions intended for a supportive work-family culture", Evidence-Based Positive Psychological Interventions in Multi-Cultural Contexts, Springer, Cham, pp. 83-103.

Lapierre, L.M., Li, Y., Kwan, H.K., Greenhaus, J.H., DiRenzo, M.S. and Shao, P. (2018), "A meta-analysis of the antecedents of work-family enrichment", Journal of Organizational Behavior, Vol. 39 No. 4, pp. 385-401.

Leonardi, C., Sabatucci, L., Susi, A. and Zancanaro, M. (2011), "Design as intercultural dialogue: coupling human-centered design with requirement engineering methods", IFIP Conference on HumanComputer Interaction, pp. 485-502.

Leonardi, C., Schiavo, G. and Zancanaro, M. (2019), "Sharing the office, sharing the care? Designing for digitally-mediated collaborative childcare in the workplace", Proceedings of the 9th International Conference on Communities \& Technologies-Transforming Communities, pp. 239-249.

Leonardi, P.M. (2012), "Materiality, sociomateriality, and socio-technical systems: what do these terms mean? How are they different? Do We need them?", in Leonardi, P.M., Nardi, B.A. and Kallinikos, J. (Eds), Materiality and Organizing, Oxford University Press, UK, pp. 24-48.

Madanipour, A., Knierbein, S. and Degros, A. (Eds). (2013), Public Space and the Challenges of Urban Transformation in Europe, Routledge, UK.

Manzini, E. and Rizzo, F. (2011), "Small projects/large changes: participatory design as an open participated process", CoDesign, Vol. 7 Nos 3/4, pp. 199-215.

Mulder, I. and Stappers, P.J. (2009), "Co-creating in practice: results and challenges", IEEE International Technology Management Conference (ICE), pp. 1-8.

Passani, A., Spagnoli, F., Bellini, F., Prampolini, A. and Firus, K. (2016), "Collective awareness platform for sustainability and social innovation (CAPS)", Organizational Innovation and Change, Springer, Cham, pp. 103-114.

Patton, Q.M. (2014), Qualitative Research and Evaluation Methods, 4th ed., Sage Publishing, UK.

Rutter, J. (2016), "Innovation in childcare. NESTA and the Family and Childcare Trust”, London.

Schiavo, G., Leonardi, C. and Zancanaro, M. (2020), "Values and practices behind collaborative childcare in knowledge-based organizations", Technology Innovation Management Review, Vol. 10 No. 5, pp. 41-50.

Silverman, D. (Ed.) (2016), Qualitative Research, 4th ed., Sage Publishing, UK.

Sousa, M.C.D. and Dierendonck, D.V. (2010), "Knowledge workers, servant leadership and the search for meaning in knowledge-driven organizations", On the Horizon - The Strategic Planning Resource for Education Professionals, Vol. 18 No. 3, pp. 230-239.

Tchounikine, P. (2017), "Designing for appropriation: a theoretical account", Human-Computer Interaction, Vol. 32 No. 4, pp. 155-195, doi: 10.1080/07370024.2016.1203263.

UNICEF Research Office. (2014), "Children of the Recession The impact of the economic crisis on child well-being in rich countries", Innocenti Report Card 12, UNICEF Office of Research, Florence.

Verhoeven, I. and Tonkens, E. (2013), "Talking active citizenship: framing welfare state reform in England and The Netherlands", Social Policy and Society, Vol. 12 No. 3, pp. 415-426.

Voorberg, W.H., Bekkers, V.J. and Tummers, L.G. (2015), "A systematic review of co-creation and co-production: embarking on the social innovation journey", Public Management Review, Vol. 17 No. 9, pp. 1333-1357.

Wong, K., Chan, A.H.S. and Teh, P.-L. (2020), "How is work-life balance arrangement associated with organisational performance? A meta-analysis", International Journal of Environmental Research and Public Health, Vol. 17 No. 12, p. 4446

Wulf, V., Pipek, V., Randall, D., Rohde, M., Schmidt, K. and Stevens, G. (Eds), (2018), Socio-Informatics, Oxford University Press, UK. 


\section{Further reading}

Bhardwaj, P. and Kumar, D. (2018), "A review paper on MERN stack for web development international", Journal of Innovative Research in Computer and Communication Engineering, Vol. 6, pp. 3615-3617.

Candiello, A., Albarelli, A. and Cortesi, A. (2012), "Quality and impact monitoring for local eGovernment services", Transforming Government: People, Process and Policy, Vol. 6 No. 1, pp. 112-125.

Christensen, J.H. (2009), "Using RESTful web-services and cloud computing to create next generation mobile applications", Proceedings of the 24th ACM SIGPLAN conference companion on Object oriented programming systems languages and applications, pp. 627-634.

Huynh, M.Q., Ghimire, P. and Truong, D. (2017), "Hybrid app approach: could it mark the end of native app domination?", Issues in Informing Science and Information Technology, Vol. 14, pp. 49-65.

Leonardi, C., Massa, P. and Zancanaro, M. (2014), "I'm here with my kids: investigating location sharing preferences of parents with young children", CHI'14 Extended Abstracts on Human Factors in Computing Systems, pp. 2137-2142.

Moulaert, F., MacCallum, D., Mehmood, A. and Hamdouch, A. (2013), "The international handbook on social innovation", Collective Action, Social Learning and Transdisciplinary Research, Edward Elgar, Cheltenham.

Rana, N.P., Weerakkody, V., Dwivedi, Y.K. and Piercy, N.C. (2014), "Profiling existing research on social innovation in the public sector", Information Systems Management, Vol. 31 No. 3, pp. 259-273.

Richardson, L. and Ruby, S. (2008), RESTful Web Services, O'Reilly Media, California.

Rosson, M.B. and Carroll, J.M. (2002), "Scenario-based design”, The Human-Computer Interaction Handbook: Fundamentals, Evolving Technologies and Emerging Applications, Lawrence Erlbaum Associates, New Jersey.

\section{Author affiliations}

Agostino Cortesi is based at the Department of Environmental Sciences, Statistics and Informatics, Ca'Foscari University, Venice, Italy.

Carlotta Berionni is based at the Department of Environmental Sciences, Statistics and Informatics, Ca'Foscari University, Venice, Italy.

Carina Veckman is based at the Department IMEC, Vrije Universiteit Brussels, Belgium.

Chiara Leonardi is based at FBK, Fondazione Bruno Kessler, Trento, Italy.

Gianluca Schiavo is based at FBK, Fondazione Bruno Kessler, Trento, Italy.

Massimo Zancanaro is based at the Department of Psychology and Cognitive Sciences, University of Trento, Italy.

Marzia Cescon is based at SmartVenice,Venice, Italy.

Maria Sangiuliano is based at SmartVenice,Venice, Italy.

Dimitris Tampakis is based at Vilabs Ltd., Paphos, Cyprus.

Manolis Falelakis is based at Vilabs Ltd., Paphos, Cyprus.

\section{Corresponding author}

Agostino Cortesi can be contacted at: cortesi@unive.it

For instructions on how to order reprints of this article, please visit our website: www.emeraldgrouppublishing.com/licensing/reprints.htm

Or contact us for further details: permissions@emeraldinsight.com 